\title{
Asparaginyl endopeptidase enhances pancreatic ductal adenocarcinoma cell invasion in an exosome-dependent manner and correlates with poor prognosis
}

\author{
QIANG YAN，WEN-BIN YUAN，XU SUN，MING-JIE ZHANG，FENG CEN，SHI-YU ZHOU, \\ WAN-BO WU, YONG-CAN XU, LI-HUI TONG and ZHI-HONG MA \\ Department of General Surgery, Huzhou Central Hospital, Huzhou, Zhejiang 313003, P.R. China
}

Received July 31, 2017; Accepted March 7, 2018

DOI: $10.3892 /$ ijo. 2018.4318

\begin{abstract}
Pancreatic cancer is one of the most lethal types of cancer; owing to low early detection rates and high metastasis rates, it is associated with an extremely poor prognosis. Therefore, a better understanding of the molecular mechanisms that underlie its metastasis and the identification of potential prognostic biomarkers are urgently required. Although high expression levels of asparaginyl endopeptidase (AEP) have been detected in various types of solid tumor, the expression and functions of AEP in pancreatic carcinomas have yet to be determined. The present study aimed to examine the putative functions of AEP in pancreatic carcinoma. Immunohistochemical analysis revealed that AEP was highly expressed in pancreatic cancer tissues compared with adjacent normal tissues. Patients with high AEP expression exhibited a significantly shorter overall survival time. Results from multivariate Cox regression analysis revealed that AEP was an independent prognostic factor for overall survival. Gain- and loss-of-function experiments demonstrated that knockdown of AEP expression significantly reduced the invasive ability of pancreatic cancer cells, whereas overexpression of AEP increased the invasive ability. In addition, AEP was detected in exosomes that were derived from cultured pancreatic ductal adenocarcinoma cells (PDACs) and in the serum from patients with PDAC. The Matrigel-Transwell invasion assay revealed that exosomes enriched with AEP were able to enhance the invasive ability of PDAC cells, whereas exosomes lacking AEP decreased the invasive ability. Furthermore, results from the present study suggested that AEP may be crucial for activation of the phosphoinositide 3-kinase/RAC- $\alpha$ serine/ threonine-protein kinase signaling pathway in PDAC cells.
\end{abstract}

Correspondence to: Professor Qiang Yan, Department of General Surgery, Huzhou Central Hospital, 198 Hongqi Road, Huzhou, Zhejiang 313003, P.R. China

E-mail: 1837803391@qq.com

Key words: pancreatic carcinoma, asparaginyl endopeptidase, exosome, invasion, RAC- $\alpha$ serine/threonine-protein kinase
The present study data indicated that high AEP expression may be important for pancreatic carcinoma progression in an exosome-dependent manner, and that AEP may be an independent indicator of poor prognosis in patients with PDAC and may be a novel prognostic biomarker or therapeutic target in pancreatic carcinoma.

\section{Introduction}

Pancreatic adenocarcinoma is one of the most common types of cancers (1). As the symptoms of pancreatic adenocarcinoma are generally non-specific, early diagnostic rates are extremely low; as such, pancreatic adenocarcinoma is often detected at an advanced stage with extensive metastasis, and has a poor prognosis $(2,3)$. The median survival time of pancreatic adenocarcinoma is 8-12 months for patients with locally advanced disease, and 3-6 months for patients with metastases (4). Pancreatic ductal adenocarcinoma (PDAC) is the most common type of pancreatic cancer and is the fourth leading cause of cancer-related mortality, with a 5-year overall survival (OS) rate for patients with metastatic PDAC at $8 \%$, which is the lowest OS rate among all types of cancer $(5,6)$. Although new therapies have been introduced, there has not been a notable improvement in OS rates for patients with PDAC (7). Thus, there is an urgent need to elucidate the underlying mechanisms of pancreatic cancer metastasis.

Asparaginyl endopeptidase (AEP; also known as legumain) is a member of the C13 family of cysteine proteases; it specifically hydrolyzes carboxy-terminally to asparagine (8). AEP occurs in acidic endosomes and lysosomes, and participates in intracellular protein degradation under physiological conditions (9). AEP was reported to function in kidney physiology (10), immunity (11) and osteoclast formation (12). High AEP expression levels have been identified in certain solid tumors, including colorectal cancer and breast cancer, and high AEP expression was previously reported to correlate with a more metastatic phenotype, which was partially due to the activation of cathepsin proteases and pro-protein matrix metalloproteinase 2 (13-16). A previous study reported that AEP exhibited a vesicular staining pattern, and the expression of AEP was significantly related to advanced tumor stage, high Gleason score, perineural invasion and larger tumor size 
in patients with prostate cancer (17). However, whether AEP participates in pancreatic cancer metastasis remains unknown.

Exosomes are nanosized membrane vesicles, with a diameter between 30 and $100 \mathrm{~nm}$, which are generated from endosomal compartment invaginations (18-20). As reported previously, tumor cell-derived exosomes serve important roles in regulating certain functions, such as cell proliferation, invasion and angiogenesis, by effectively delivering microRNAs, mRNAs and proteins to other cells (21-23). However, the functions and underlying mechanisms of exosomes secreted by pancreatic cancer cells remains unknown.

Pancreatic cancer cell survival is often due to survivalpromoting signals, including increased expression of apoptosis regulator $\mathrm{BCL}-2$ and activation of phosphoinositide 3-kinase $(\mathrm{PI} 3 \mathrm{~K}) / \mathrm{RAC}-\alpha$ serine/threonine-protein kinase (AKT) signaling (24-26), both of which have been associated with pancreatic adenocarcinoma progression in human tissues and in animal models. Activation of the PI3K/AKT pathway due to gene amplification, activating mutations or loss of suppressors has been reported in several types of human cancer, such as colorectal, lung, cervical, gastric and pancreatic cancer (27-29).

\section{Materials and methods}

Patients and tissue samples. The present study was approved by the Ethics Committee of Huzhou Central Hospital, Zhejiang University (Huzhou, China). Written informed consent was obtained from patients, or from the guardians on behalf of the minors, prior to enrollment in the present study. Patient diagnoses were independently reviewed by two pathologists and classified according to the WHO criteria. A total of 63 patients (age range, 43-85 years) with histologically confirmed PDAC that were treated at Huzhou Central Hospital of Zhejiang University were recruited for this study between May 2009 and December 2014. Of the 63 patient samples collected, 6 were paired fresh PDAC tissues and adjacent normal tissues. Follow-up data were available for all 63 patients. Sera were also collected from three patients that suffered pancreatitis and three patients with PDAC.

Cell lines. The human PDAC cell lines PANC-1 (catalog no. TCHu98), BxPC3 (catalog no. TCHu12) and ASPC-1 (catalog no. TCHu8) were purchased from the Cell Bank of Type Culture Collection of the Chinese Academy of Sciences (Shanghai, China). Capan-1 was purchased from the American Type Culture Collection (catalog no. HTB-79; Manassas, VA, USA). Cells were maintained in Dulbecco's modified Eagle's medium supplemented with $10 \%$ heat-inactivated fetal bovine serum (FBS; Invitrogen; Thermo Fisher Scientific, Inc., Waltham, MA, USA), penicillin (100 U/ml) and streptomycin $(100 \mu \mathrm{g} / \mathrm{ml})$ at $37^{\circ} \mathrm{C}$ in a humidified atmosphere of $5 \% \mathrm{CO}_{2}$. All cells were free of mycoplasma contamination.

Plasmids and reagents. Lentiviral vectors for AEP knockdown (KD) or overexpression (OE) were constructed by Shanghai Hanyin Biotechnology Co., Ltd. (Shanghai, China). Empty vector was used as negative control (NC) for AEP-KD and -OE experiments; AEP-targeted KD sequences and AEP-OE sequences were used as previously described (30). AEP-targeted KD sequences were: KD1, 5'-GATGGTGTT
CTACATTGAA-3', and KD2, 5'-GGGGACTGGTACAGCG TCA-3'. The lentiviral particles were packaged using psPAX2 and pMD2G plasmids (Shanghai Hanyin Biotechnology Co., Ltd.). To obtain stable cells with reduced or overexpressed AEP, lentivirus-containing supernatants (Shanghai Hanyin Biotechnology Co., Ltd.) were added to the PDAC cells, followed by selection with $1 \mu \mathrm{g} / \mathrm{ml}$ puromycin (Shanghai Hanyin Biotechnology Co., Ltd.) for 2 weeks to select stably expressing AEP-KD1, AEP-KD2 or AEP-OE cells (31).

Primary antibodies used in the present study included: Goat anti-human AEP (catalog no. AF2199; R\&D Systems; Bio-Techne, Abingdon, UK), rabbit anti-human AKT (catalog no. 4685; Cell Signaling Technology, Inc., Danvers, MA, USA), rabbit anti-human phosphorylated (p)-AKT (catalog no. 4060; Cell Signaling Technology, Inc.), rabbit anti-CD63 (catalog no. ab68418; Abcam, Cambridge, UK), rabbit anti-human PI3K (catalog no. 3811; Cell Signaling Technology, Inc.), and rabbit anti- $\beta$-actin (catalog no. ab8227; Abcam); and the horseradish peroxidase (HRP)-conjugated donkey anti-goat immunoglobulin G (IgG; catalog no. 705-036-147; Jackson ImmunoResearch, Inc.) the horseradish peroxidase (HRP)-conjugated goat anti-rabbit IgG (catalog no. 7074; Cell Signaling Technology, Inc.) secondary antibody was also used in the present study.

Immunohistochemical analysis. Tissues were fixed in $4 \%$ paraformaldehyde overnight at $4^{\circ} \mathrm{C}$, embedded in paraffin and sectioned $(6 \mu \mathrm{m})$. Immunohistochemical analyses were performed as previously described (32). Goat anti-human AEP antibody (catalog no. AF2199; R\&D Systems; Bio-Techne, Abingdon, UK; diluted 1:200 in blocking buffer) was used as primary antibody. Biotin-conjugated donkey anti-goat IgG (catalog no. 705-066-147; Jackson ImmunoResearch, Inc.) was used as secondary antibody. Normal goat IgG (catalog no. AB-108-C; R\&D Systems; Bio-Techne) was included as negative control. The proportion of positive protein expressions were evaluated as follows: A score of 0 was indicated if $0 \%$ of the tumor cells showed positive staining; 1 if $0-10 \%$ of cells were stained; $2,11-50 \%$ stained; $3,51-75 \%$ stained; and 4 if $75-100 \%$ stained. The intensity of staining was rated on a scale of 0 to 3 : 0 , negative; 1 , weak; 2 , moderate; and 3 , strong. The proportion and intensity scores were combined to obtain a total score (range 0-6) and designated 0-3.5 as low expression and 4-6 as high expression.

Western blot analysis. Total protein was extracted from cells, exosomes and tissue samples using RIPA lysis and extraction buffer (Thermo Fisher Scientific, Inc.). Protein concentration was determined using the bicinchoninic acid protein assay method. Lysates ( $50 \mu \mathrm{g}$ per lane) were separated by $8 \%$ SDS-PAGE and transferred to nitrocellulose membranes. Membranes were blocked with $5 \%$ non-fat milk for $30 \mathrm{~min}$ at $25^{\circ} \mathrm{C}$, followed by overnight incubation with primary antibodies (1:500) at $4^{\circ} \mathrm{C}$. Subsequently, membranes were incubated with HRP-conjugated secondary antibodies $(1: 3,000)$ for $60 \mathrm{~min}$ at $25^{\circ} \mathrm{C}$. Immunoreactive proteins were visualized with the Immobilon Western Chemiluminescent HRP Substrate (cat. no. WBKLS0500; EMD Millipore, Billerica, MA, USA). Quantity One analysis software version 4.6.9 (Bio-Rad Laboratories, Inc., Hercules, CA, USA) was used 
to quantify the relative band intensities from western blotting images; actin or CD63 was used for loading controls and for normalization. The assays were conducted in triplicate.

Total RNA isolation and reverse transcription-quantitative polymerase chain reaction $(R T-q P C R)$ analysis. Total RNA was extracted from PDAC cells $\left(2 \times 10^{6}\right)$ using TRIzol reagent (Invitrogen; Thermo Fisher Scientific, Inc.), according to the manufacturer's instructions. cDNA was reverse transcribed from $1 \mu \mathrm{g}$ total RNA using the Promega Reverse Transcription System (cat no. A3500; Promega Corporation, Madison, WI, USA). qPCR was performed with the SYBR Premix Ex Taq kit (Takara Biotechnology Co., Ltd., Dalian, China). Primers were purchased from Sangon Biotech Co., Ltd. (Shanghai, China), and the sequences are as follows: AEP, forward 5'-TCA GGGTATGAAACGCAAAGC-3', reverse 5'-GAGACGATCT TACGCACTGAC-3'; GAPDH, forward 5'-CATGGCCTTCC GTGTTCCTA-3', reverse 5'-GCGGCACGTCAGATCCA-3'; GAPDH was used as a loading control. Thermocycling conditions comprised initial denaturation at $95^{\circ} \mathrm{C}(5 \mathrm{~min})$, followed by 36 cycles of denaturation at $95^{\circ} \mathrm{C}(10 \mathrm{sec})$ and annealing/ elongation at $60^{\circ} \mathrm{C}(30 \mathrm{sec})$. Relative mRNA expression levels were calculated using the $2^{-\Delta \Delta \mathrm{Cq}}$ method using the housekeeping gene GAPDH for normalization (33). The assays were conducted in triplicate.

Exosome isolation and culture method. To isolate exosomes, PDAC cells were cultured for $48 \mathrm{~h}$ at $37^{\circ} \mathrm{C}$ and the supernatants of these cells were collected and centrifuged twice $\left(1,000 \mathrm{x} \mathrm{g}\right.$ for $10 \mathrm{~min}$ at $4^{\circ} \mathrm{C}$, and $3,000 \mathrm{x} \mathrm{g}$ for $30 \mathrm{~min}$ at $\left.4^{\circ} \mathrm{C}\right)$ to remove cells and fragments. Subsequently, the exosome isolation reagent from the Total Exosome Isolation kit (Invitrogen; Thermo Fisher Scientific, Inc.) was added to the cell media sample and incubated overnight at $4^{\circ} \mathrm{C}$. The precipitated exosomes were recovered by centrifugation at $10,000 \mathrm{x}$ for $1 \mathrm{~h}$ at $4^{\circ} \mathrm{C}$. For exosome isolation in sera, the ExoQuick Exosome Precipitation Solution (catalog no. EXOQ5A-1; System Biosciences, Palo Alto, CA, USA) was used to isolate exosomes from serum samples, according to the manufacturer's instructions. Exosomes were re-suspended in PBS and stored at $-80^{\circ} \mathrm{C}$. The concentration of exosomes was determined by BCA protein assay. Exosomes $(50 \mathrm{ng} / \mu \mathrm{l})$ were added to $1 \times 10^{5}$ cells in culture medium for $24 \mathrm{~h}$ at $37^{\circ} \mathrm{C}$, as previously described (34). The assays were conducted in triplicate.

Transmission electron microscopy. The exosome suspension was added to an equal volume of $4 \%$ paraformaldehyde at $4^{\circ} \mathrm{C}$ for $30 \mathrm{~min}$ and applied to a Formvar/Carbon film-coated transmission electron microscope grid (Alliance Biosystems, Inc., Osaka, Japan). Subsequently, the sample was fixed by incubation with $1 \%$ glutaraldehyde for $5 \mathrm{~min}$ at $25^{\circ} \mathrm{C}$, washed with PBS and contrasted with $1 \%$ uranyl acetate for 5 min at $25^{\circ} \mathrm{C}$. Samples were embedded in epoxy resin and polymerized at $35^{\circ} \mathrm{C}$ for $12 \mathrm{~h}, 45^{\circ} \mathrm{C}$ for $12 \mathrm{~h}$ and $60^{\circ} \mathrm{C}$ for $24 \mathrm{~h}$. Exosomes were subsequently observed under a Hitachi H-7650 transmission electron microscope (Hitachi, Ltd., Tokyo, Japan).

Cell invasion assay. Cells $\left(1 \times 10^{5}\right)$ were seeded into the upper chambers of Matrigel-coated Transwell chambers (pore size,
$8 \mu \mathrm{m}$ ) in serum-free DMEM. DMEM containing 10\% FBS was added to the lower chamber as a chemoattractant. Following incubation for $24 \mathrm{~h}$ at $37^{\circ} \mathrm{C}$, the upper surfaces of the inserts were gently wiped with a cotton swab and cells that had invaded the lower chambers were fixed with $4 \%$ paraformaldehyde and stained with $0.1 \%$ crystal violet at $37^{\circ} \mathrm{C}$ for $30 \mathrm{~min}$. The number of invading cells was counted under an Olympus CKX41 inverted microscope (Olympus Corporation, Tokyo, Japan); five random microscopic fields were analyzed for each insert. The assays were conducted in triplicate.

Statistical analysis. OS rates were calculated from the date of surgery to the date of death or last follow-up; survival curves were plotted using the Kaplan-Meier method and compared using the log-rank test. Median survival times and hazard ratios (HRs) were shown with 95\% confidence intervals (CIs). Data are presented as the mean \pm standard deviation. To assess the differences between groups, categorical variables were compared by means of $\chi^{2}$ analysis. Analysis of variance tests were followed by two-tailed Dunn's post-hoc analysis or Tukey's multiple comparisons test to identify statistically significant differences. Statistical analyses were performed using SPSS software version 15.0 (Chicago, IL, USA). $\mathrm{P}<0.05$ was considered to indicate a statistically significant difference.

\section{Results}

AEP expression in human PDAC tissues. AEP protein expression levels were analyzed in freshly collected human PDAC tissues $(n=6)$ and adjacent normal tissues $(n=6)$ by western blotting (Fig. 1A). AEP expression levels were notably higher in PDAC tissues compared with expression levels in adjacent normal tissues (Fig. 1A). AEP protein expression levels were also examined by immunohistochemical analysis in the 6 matched tissues as well as the remaining 57 tumoral tissues (Fig. 1B and C, respectively). Consistent with the western blotting results, AEP staining was stronger in tumoral tissues compared with expression levels in the adjacent normal tissues (Fig. 1B). The staining of AEP was revealed to be mainly localized in the cytoplasm in the PDAC tissues (Fig. 1B and C).

Relationship between AEP expression and the clinicopathological features of patients with PDAC. According to the expression level score of AEP protein in PDAC samples, all cases were distributed into two groups: A low AEP expression group $(n=35)$, and a high AEP expression group ( $n=28$; Fig. 1C; Table I). Following evaluation of the immunohistochemical staining results, AEP staining levels in the American Joint Committee on Cancer (AJCC) stage II cases were significantly higher compared with staining level in the AJCC stage I cases $(\mathrm{P}=0.009$; Table I). The expression of AEP in PDAC tissues exhibited a strong association with AJCC stage, although no associations were found between AEP expression and other clinicopathological features (Table I).

AEP expression and patient prognosis. To assess the relationship between the level of AEP expression with patient prognosis the Kaplan-Meier and log-rank tests were used to evaluate the effects of AEP expression on patient OS. Patients 
A
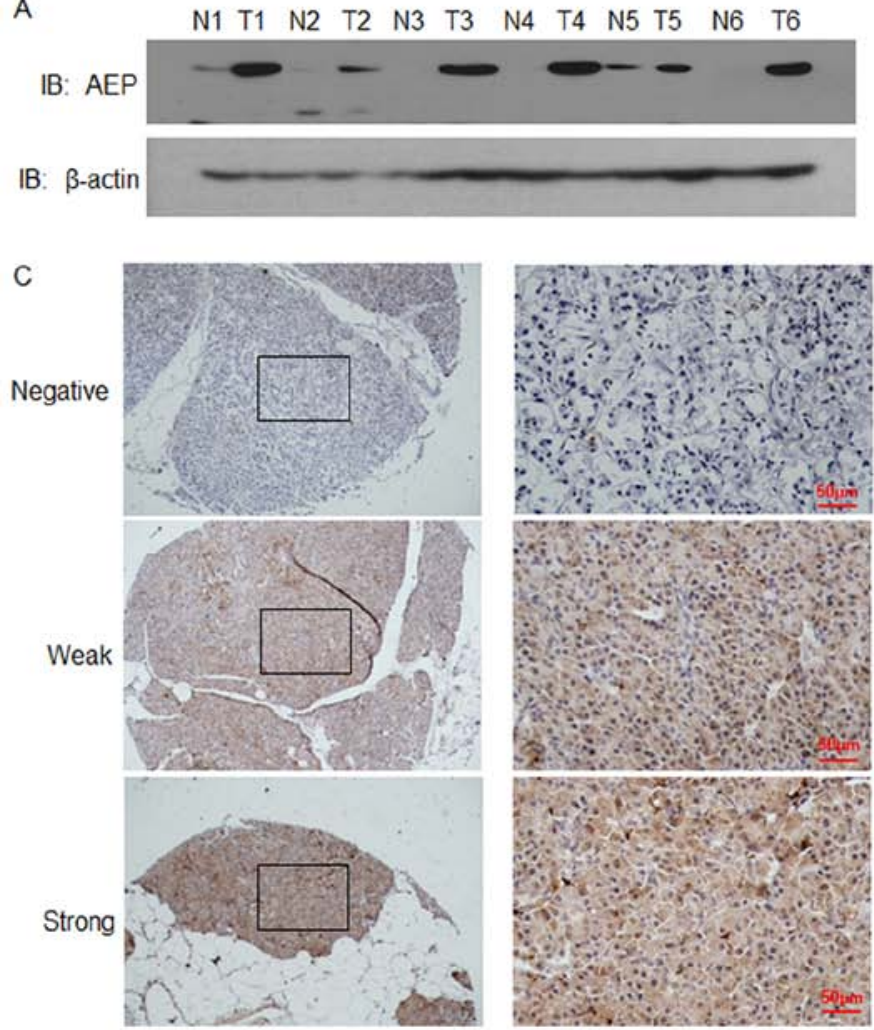

B

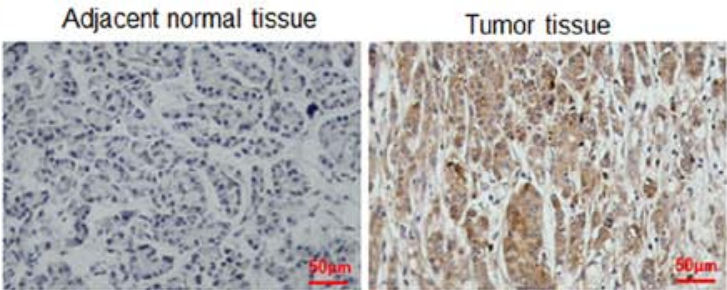

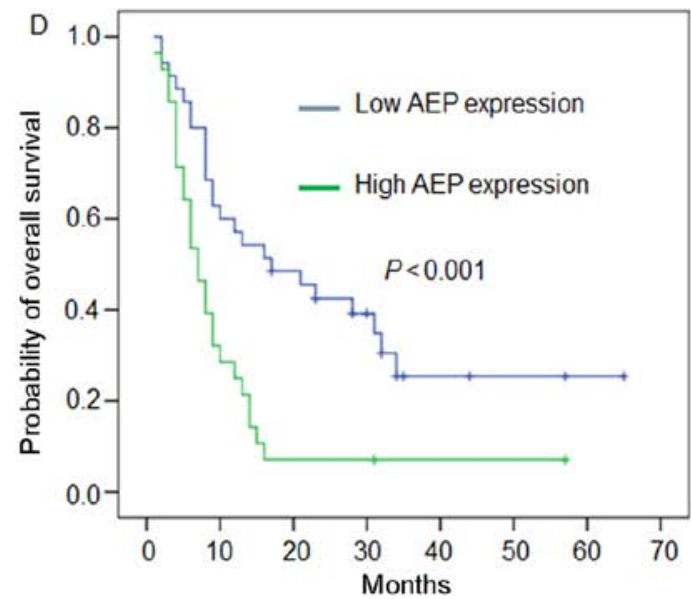

Figure 1. High AEP expression levels in pancreatic cancer tissues predicts poor prognosis. (A) Western blot analysis of AEP protein expression in pancreatic adenocarcinoma tissues $(n=6)$ and adjacent normal tissues $(n=6)$. (B) Immunohistochemical analysis of AEP expression in pancreatic adenocarcinoma tissues and adjacent normal tissues; magnification: left, x40; right x200. (C) Immunohistochemical analysis of AEP in pancreatic adenocarcinoma tissues. Representative images of pancreatic adenocarcinoma tissues exhibiting negative, weak and strong AEP staining. (D) Kaplan-Meier curves of overall survival in high and low AEP expression groups. AEP, asparaginyl endopeptidase; $\mathrm{N}$, normal adjacent tissue; $\mathrm{T}$, pancreatic adenocarcinoma tissue.

Table I. Association of AEP expression with clinicopathological variables in pancreatic ductal adenocarcinoma.

\begin{tabular}{lcccc}
\hline & & \multicolumn{2}{c}{ AEP staining (n; \%) } & \\
$\begin{array}{l}\text { Clinicopathological } \\
\text { characteristic }\end{array}$ & $\mathrm{n}(\%)$ & Low & High & P-value \\
\hline Age (year) & & & & \\
$<60$ & & & & 0.599 \\
$\geq 60$ & $22(34.92)$ & $11(50.00)$ & $11(50.00)$ & \\
Sex & $41(60.08)$ & $24(58.54)$ & $17(41.46)$ & \\
Male & & & & 0.798 \\
Female & $27(42.86)$ & $16(59.26)$ & $11(40.74)$ & \\
AJCC stage & $36(57.14)$ & $19(52.78)$ & $17(47.22)$ & \\
I & & & & $\mathbf{0 . 0 0 9}$ \\
II & $7(11.11)$ & $6(85.71)$ & $1(14.29)$ & \\
Tumor location & $56(88.89)$ & $29(51.79)$ & $27(48.21)$ & \\
$\quad$ Head & $37(58.73)$ & $18(48.65)$ & $19(51.35)$ & 0.209 \\
Body/tail & $26(41.27)$ & $17(65.38)$ & $9(34.62)$ & \\
& & &
\end{tabular}

AEP, asparaginyl endopeptidase; AJCC, American Joint Committee on Cancer.

with a high level of AEP expression in tumoral tissues had significantly shorter OS times compared with patients with low AEP expression $(n=63 ; P=0.005$; Fig. 1D and Table II).
Table II. Median for survival time with 95\% CI and the log-rank test.

\begin{tabular}{lccrcr}
\hline $\begin{array}{l}\text { AEP } \\
\text { expression }\end{array}$ & $\mathrm{n}$ & $\begin{array}{c}\text { Mean } \\
\text { (months) }\end{array}$ & $95 \%$ CI & $\begin{array}{c}\chi^{2} \\
\text { (log-rank) }\end{array}$ & P-value \\
\hline Low & 35 & 20.29 & $14.95-25.63$ & & \\
High & 28 & 10.11 & $5.84-14.37$ & 2.536 & $\mathbf{0 . 0 0 5}$ \\
Overall & 63 & 15.76 & $12.10-19.42$ & & \\
\hline
\end{tabular}

AEP, asparaginyl endopeptidase; CI, confidence interval.

The mean OS time of patients with low AEP expression was 20.29 months $(n=35 ; 95 \%$ CI, 14.95-25.63; Table II), whereas the OS time of patients with high AEP expression was 10.11 months $(n=28 ; 95 \%$ CI, 5.84-14.37). The log-rank test (univariate analysis) revealed that the patients with low AEP expression had a longer OS time $\left(\chi^{2}=2.536 ; \mathrm{P}=0.005\right.$; Table II). Furthermore, multivariate Cox regression analysis was also performed, which indicated that AEP expression was an independent prognostic factor $(\mathrm{HR}=2.415 ; 95 \% \mathrm{CI}$, 1.345-4.334; $\mathrm{P}=0.003$; Table III), whereas AJCC stage was not $(\mathrm{HR}=1.475 ; 95 \% \mathrm{CI}, 0.574-3.787 ; \mathrm{P}=0.419)$.

AEP enhances PDAC cell invasive ability. To examine the functions of AEP in pancreatic adenocarcinoma progression, 

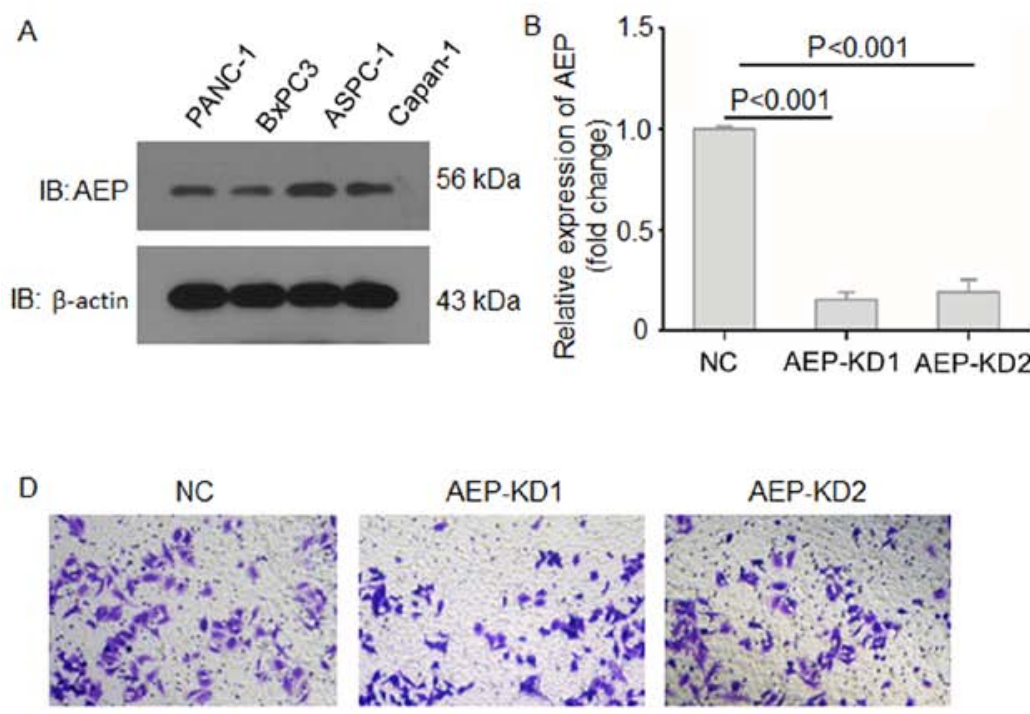

C
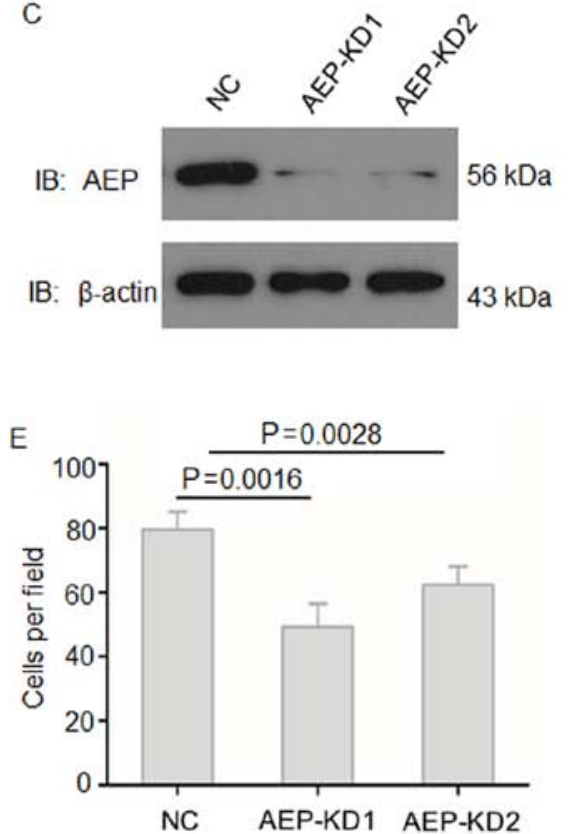

Figure 2. Suppression of AEP expression inhibits the invasive ability of PDAC cells. (A) Western blot analysis of AEP protein expression levels in several PDAC cell lines. (B) Results from reverse transcription-quantitative polymerase chain reaction analysis of AEP mRNA expression in ASPC-1 cells with or without AEP knockdown. (C) Western blotting results of AEP protein expression in ASPC-1 cells with and without AEP knockdown. (D and E) MatrigelTranswell invasion analysis of ASPC-1 cells with or without AEP knockdown. The invasive ability of ASPC-1 cells with AEP knockdown was significantly reduced compared with that of the $\mathrm{NC}$ group. Representative staining images are shown; magnification $\mathrm{x} 200$. AEP, asparaginyl endopeptidase; NC, negative control; KD, knockdown; PDAC, pancreatic ductal adenocarcinoma.

Table III. Univariate and multivariate analysis of overall survival for patients with pancreatic ductal adenocarcinoma.

\begin{tabular}{|c|c|c|c|c|}
\hline \multirow[b]{3}{*}{$\begin{array}{l}\text { Clinicopathological } \\
\text { characteristic }\end{array}$} & \multicolumn{4}{|c|}{ Overall survival } \\
\hline & \multicolumn{2}{|c|}{$\begin{array}{l}\text { Univariate } \\
\text { analysis }\end{array}$} & \multicolumn{2}{|c|}{$\begin{array}{c}\text { Multivariate } \\
\text { analysis }\end{array}$} \\
\hline & Mean \pm SEM & P-value ${ }^{a}$ & a HR & P-value \\
\hline \multicolumn{5}{|l|}{$\begin{array}{l}\text { AEP expression } \\
\text { in tumor tissues }\end{array}$} \\
\hline Low & $20.29 \pm 2.63$ & 0.005 & - & - \\
\hline High & $10.11 \pm 2.08$ & & 2.415 & 0.003 \\
\hline \multicolumn{5}{|l|}{ Age (year) } \\
\hline$<60$ & $16.91 \pm 2.86$ & 0.65 & - & - \\
\hline$\geq 60$ & $15.15 \pm 2.38$ & & - & - \\
\hline \multicolumn{5}{|l|}{ Sex } \\
\hline Male & $16.96 \pm 2.66$ & 0.574 & - & - \\
\hline Female & $14.86 \pm 2.53$ & & - & - \\
\hline \multicolumn{5}{|l|}{ AJCC stage } \\
\hline I & $29.86 \pm 8.45$ & 0.006 & - & - \\
\hline II & $15.76 \pm 1.83$ & & 1.475 & 0.419 \\
\hline \multicolumn{5}{|l|}{ Tumor location } \\
\hline Head & $12.81 \pm 1.65$ & 0.054 & - & - \\
\hline Body/tail & $19.76 \pm 1.83$ & & - & - \\
\hline
\end{tabular}

${ }^{a}$ Log-rank. AEP, asparaginyl endopeptidase; AJCC, American Joint Committee on Cancer; HR, hazard ratio; SEM, standard error of the mean. the expression levels of AEP protein were first examined in several PDAC cell lines by western blotting (Fig. 2A). The results showed that AEP was expressed in all PDAC cell lines. Subsequently, two AEP-KD lentiviral vectors were constructed and used to knock down AEP expression in ASPC-1 cells (Fig. 2B and C). The RT-qPCR and western blotting results demonstrated that AEP was effectively knocked down upon treatment with AEP-KD1 and -KD2 compared with NC-treated cells. The effects of AEP on the invasive ability of PDAC cells were assessed by Matrigel-Transwell invasion assay, which indicated that suppression of AEP expression resulted in reduced invasive ability of ASPC-1 cells compared with NC-treated cells (Fig. 2D and E). AEP-OE BxPC-3 cells were also constructed and verified by RT-qPCR and western blotting (Fig. 3A and B); overexpression of AEP in BxPC3 cells significantly increased the invasive ability of these cells compared with NC-treated cells (Fig. 3C and D). These data suggested that AEP may be crucial for the invasive phenotype of PDAC cells.

Secreted exosomal AEP regulates the invasive ability of $P D A C$ cells. AEP was previously reported to be a secreted protein (27), and exosomes are key mediators and modulators of cell-cell communications to promoter tumor metastasis. Therefore, the exosomes secreted by PDAC cells were collected and analyzed. The morphology of exosomes was observed under transmission electron microscopy; exosomes are round in appearance and $\sim 100 \mathrm{~nm}$ in diameter (Fig. 4A). Western blotting results demonstrated that the exosomes from each PDAC cell line expressed AEP protein (Fig. 4B). When AEP expression was knocked down in ASPC-1 cells, exosomal AEP protein expression was notably 

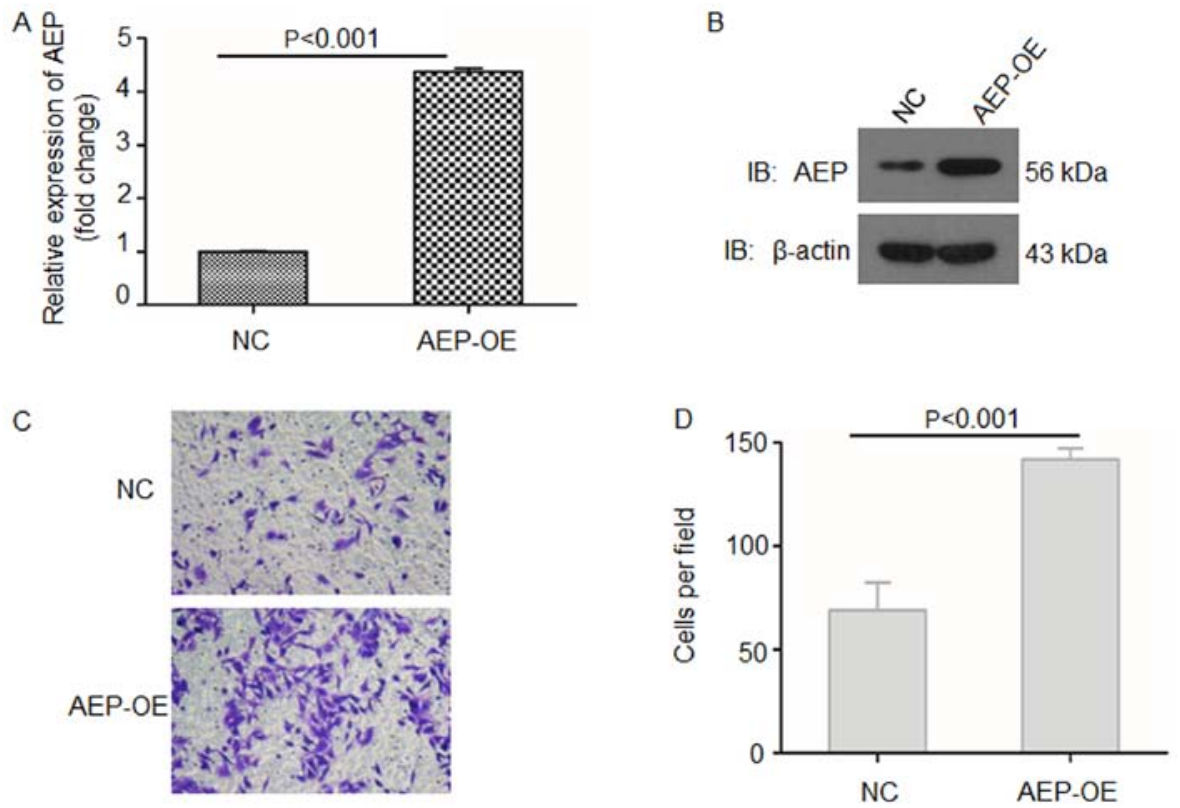

Figure 3. AEP overexpression of enhances the invasive ability of BxPC3 pancreatic ductal adenocarcinoma cells. (A) Results from reverse transcriptionquantitative polymerase chain reaction analysis of AEP mRNA expression in BxPC3 cells with or without AEP overexpression. (B) Western blotting results of AEP protein expression in BxPC3 with or without AEP overexpression. (C and D) Matrigel-Transwell invasion analysis of BxPC3 cells with or without AEP overexpression. Representative staining images are shown; magnification x200. AEP, asparaginyl endopeptidase; NC, negative control; OE, overexpression.

A

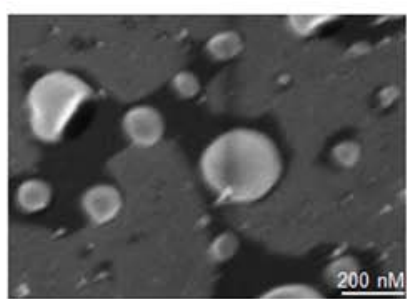

B

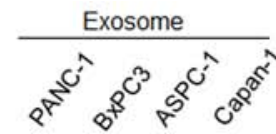

IB:AEP

IB:CD63

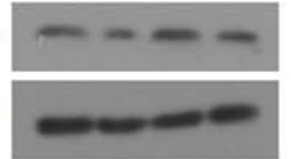

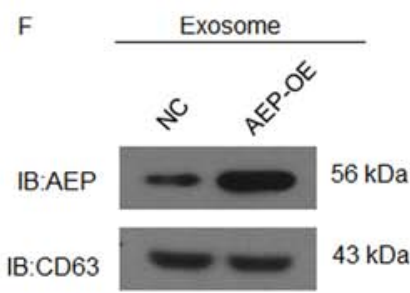

G

NC

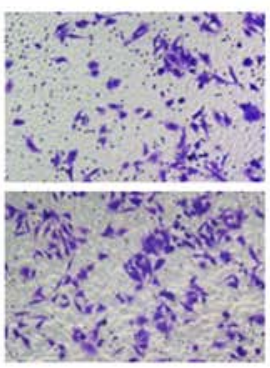

C

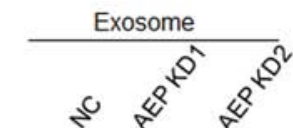

$56 \mathrm{kDa}$

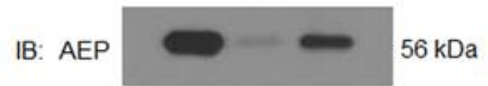

$24 \mathrm{kDa} \quad \mathrm{IB}: \mathrm{CD} 63 \mathrm{kDa}$

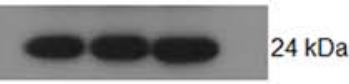

E
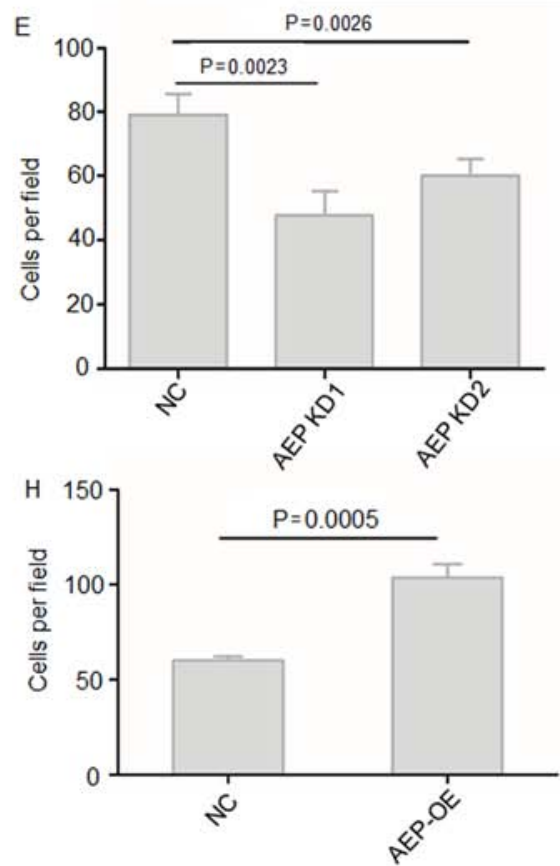

Figure 4. Secreted AEP regulates the invasive ability of PDAC cells. (A) Representative electron micrograph image of exosomes isolated from ASPC-1 cells revealing the round-shaped morphology and size of $\sim 100 \mathrm{~nm}$; scale bar, $200 \mathrm{~nm}$. (B) Western blot analysis demonstrating the expression of AEP proteins in exosomes derived from the ASPC-1 cells; CD63 was used as a loading control for exosomes. (C) Western blotting results of exosomal AEP expression in ASPC-1 cells treated with AEP-KD1 or AEP-KD2 lentiviral vector. (D and E) Matrigel-Transwell invasion analysis of ASPC-1 cells cultured with exosomes derived from AEP-KD1 or AEP-KD2 treated ASPC-1 cells; magnification x200. (F) Western blot analysis of exosomal AEP expression in BxPC3 cells treated with an AEP-OE vector. ( $\mathrm{G}$ and $\mathrm{H})$ Matrigel-Transwell invasion analysis of BxPC3 cells cultured with exosomes derived from AEP-OE- or NC-treated BxPC3 cells. AEP, asparaginyl endopeptidase; KD, knockdown; NC, negative control; OE, overexpression; PDAC, pancreatic ductal adenocarcinoma. 
A

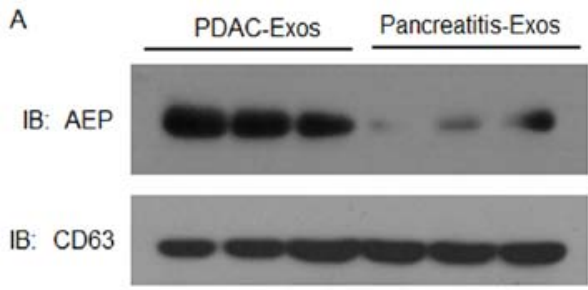

C

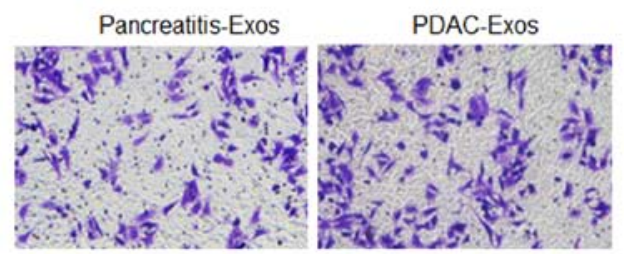

B
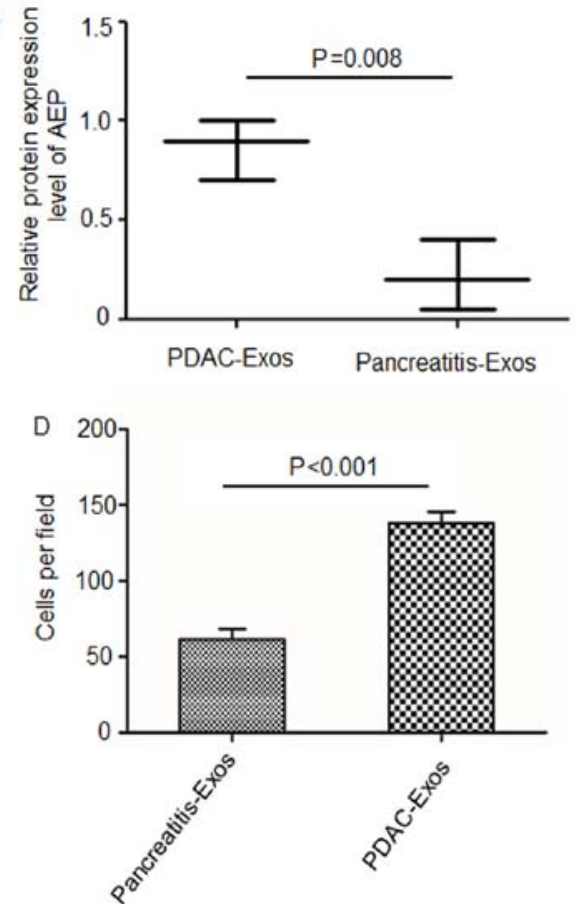

Figure 5. High levels of circulating AEP in the sera of patients with pancreatic cancer promote the invasive ability of PDAC cells. (A and B) Western blot analysis of AEP protein expression in exosomes isolated from the sera of patients with PDAC or pancreatitis; $n=3$; CD63 was used as a loading control for exosomes. The assays were conducted in triplicate. $\mathrm{P}<0.01$ vs. PDAC group. (C and D) Matrigel-Transwell invasion analysis of BxPC 3 cells cultured with the exosomes derived from the sera of patients with PDAC or pancreatitis. AEP, asparaginyl endopeptidase; exos, exosomes; PDAC, pancreatic ductal adenocarcinoma.

reduced in AEP-KD1-treatd cells compared with AEP-KD2and NC-treated cells (Fig. 4C). To further determine the putative functions of pancreatic cancer cell-derived exosomal AEP on PDAC metastasis, ASPC-1 cells were cultured with the exosomes isolated from either untreated cells or cells treated with AEP-KD1 or -KD2 and the invasive ability was examined. Results from the Matrigel-Transwell invasion assay indicated that exosomes with a low content of AEP (that is, exosomes isolated from cells treated with either AEP-KD1 or -KD2) exhibited a significantly reduced ability to promote the invasion of PDAC cells compared with cells treated with NC-exosomes (Fig. 4D and E). Conversely, exosomes derived from AEP-overexpressing BxPC3 cells significantly increased the invasive ability of treated PDAC cells compared with cells treated with NC-exosomes (Fig. 4F-H). These results suggested that exosomal AEP may be crucial for the invasive phenotype of PDAC cells.

Exosomal AEP proteins are enriched in the serum of patients with PDAC. Exosomes were isolated from the sera of patients with either PDAC or pancreatitis. Western blotting results revealed that AEP was enriched in the exosomes isolated from the sera of patients with PDAC compared with expression levels in patients with pancreatitis (Fig. 5A and B). BxPC3 cells were co-cultured with these isolated exosomes and the invasive ability was examined. Results from the Matrigel-Transwell invasion assays indicated that $\mathrm{BxPC} 3$ cells treated with exosomes collected from patients with PDAC (with a high content of AEP) exhibited a significantly higher invasive ability compared with cells treated with exosomes derived from patients with pancreatitis (Fig. 5C and D).
AEP regulates the activation of $P I 3 K / A K T$ signaling in $P D A C$ cells. PI3K/AKT signaling is an important survival pathway that is involved in carcinogenesis and malignant cell progression (22-24). Therefore, whether AEP was able to regulate the PI3K/AKT pathway in PDAC cells was investigated. Reduced AEP expression in BxPC3 cells led to decreased expression levels of p-PI3K and p-AKT, but not total PI3K or AKT expression, compared with the respective expression levels in NC-treated cells (Fig. 6A). Furthermore, AEP overexpression in BxPC3 cells resulted in significantly elevated p-PI3K and p-AKT expression levels (Fig. 6B). Cells cultured with exosomes expressing reduced levels of AEP also exhibited significantly reduced p-PI3K and p-AKT expression compared with NC-treated cells (Fig. 6C), whereas cells treated with AEP-OE exosomes exhibited increased expression levels of p-PI3K and p-AKT (Fig. 6D). These results indicated that AEP may be an important element in pancreatic cancer cell invasion and survival by regulating the PI3K/AKT pathway.

\section{Discussion}

Pancreatic cancer is a common malignancy worldwide and has a high rate of mortality (35). Therefore, the discovery of potential biomarkers and therapeutic targets is important for the improvement of clinical strategies for pancreatic adenocarcinoma. AEP is highly specific to an asparagine residue at the P1 site of its substrates (36). A study by Liu et al reported that numerous solid tumors expressed AEP, including breast cancer, colon cancer and central nervous system neoplasms (32). In addition, AEP expression was positively associated with 

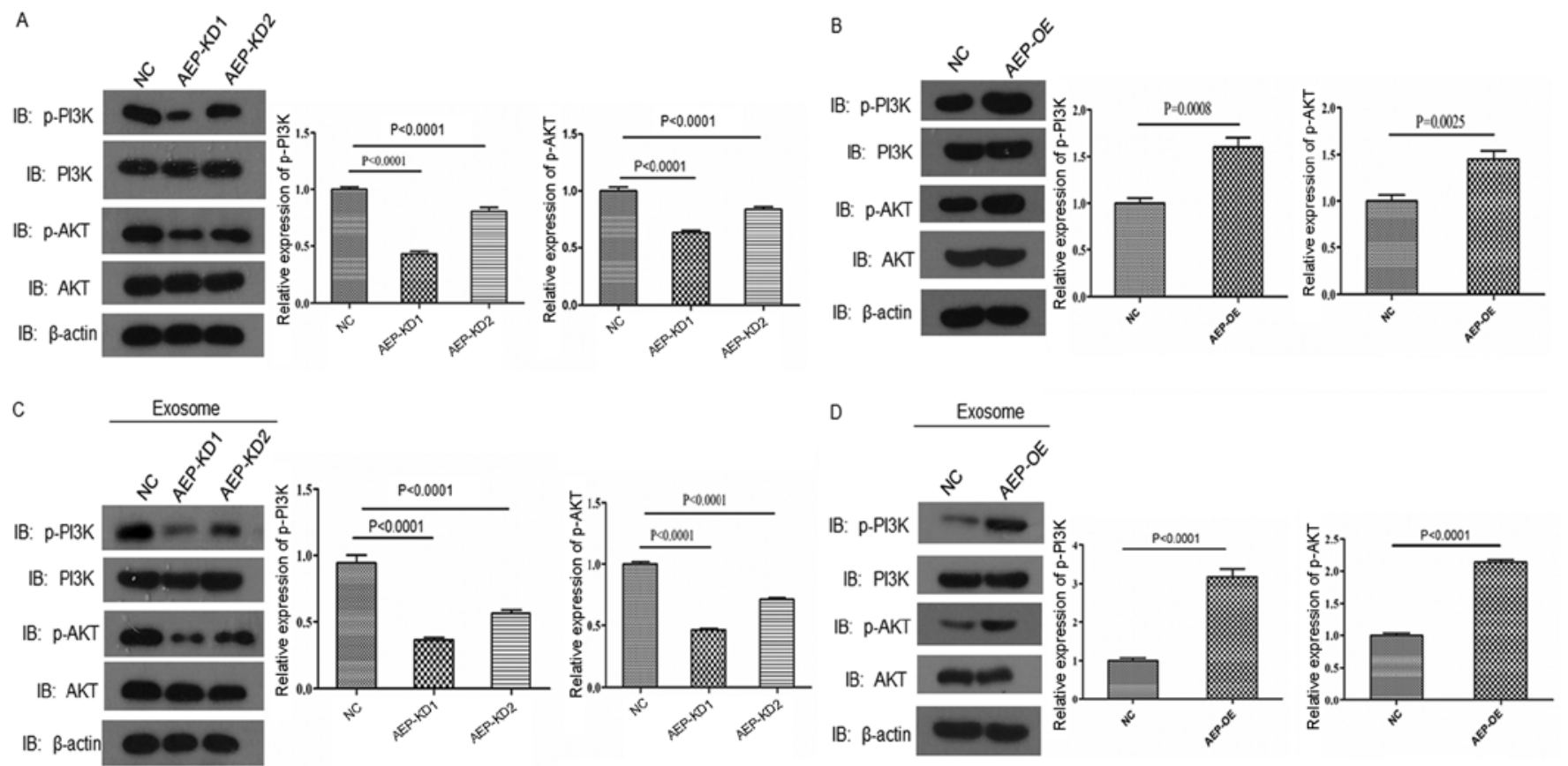

Figure 6. AEP regulates PI3K-AKT pathway activation. Western blotting results for p-PI3K, PI3K, p-AKT and AKT protein expression in (A) AEP-KD1 or -KD2-treated BxPC3 cells, (B) AEP-OE-treated BxPC3 cells, (C) BxPC3 cells cultured with exosomes derived from AEP-KD1 or -KD2-treated BxPC3 cells, or (D) BxPC3 cells cultured with exosomes derived from AEP-OE-treated BxPC3 cells. AEP, asparaginyl endopeptidase; AKT, RAC- $\alpha$ serine/threonineprotein kinase; KD, knockdown; NC, negative control; OE, overexpression; p, phosphorylated; PI3K, phosphoinositide 3-kinase.

certain clinicopathological features in patients with ovarian cancer (37) and breast cancer (14) such as stage and ascetic cytology. Although the possible involvement of AEP in several solid tumors has been reported, the present study is the first to examine the expression and function of AEP in PDAC. In the present study, AEP was demonstrated to be expressed in PDAC cell lines, and AEP was highly expressed in pancreatic cancer tissues compared with adjacent normal tissues. In addition, high AEP expression was determined to be associated with poor prognosis. Taken together, the present study results indicated that high expression of AEP was associated with pancreatic carcinoma progression and that AEP expression may independently indicate poor prognosis in patients; therefore, AEP may be a novel prognostic biomarker or therapeutic target in pancreatic carcinoma.

To date, little is known about the biological processes in which AEP may be involved in cancer progression. In the present study, gain- and loss-of-function experiments revealed that knockdown of AEP expression levels significantly reduced the invasive ability of PDAC cells, whereas overexpression of AEP increased the invasive ability. Furthermore, AEP was detected in exosomes derived from PDAC cells as well as in serum from patients with PDAC. The Matrigel-Transwell invasion assay revealed that exosomes enriched with AEP enhanced the invasive ability of PDAC cells, whereas exosomes lacking AEP decreased the invasive ability. Thus, AEP may be important for pancreatic carcinoma progression in an exosome-dependent manner. A previous study reported that AEP-containing vesicles may be found at the invasive front of a tumor, and AEP overexpression can increase cell migration and invasion (32). The present study demonstrated that the extracellular AEP-containing exosomes promoted pancreatic carcinoma cell invasive ability.
Although AEP has been reported to be an important regulator of cancer invasion and metastasis (14), the biological functions of AEP in cancer progression have not been fully investigated. Biochemical analyses revealed that AEP may be involved in pancreatic adenocarcinoma cell invasion and survival through the regulation of the PI3K-AKT pathway. A previous study reported that AEP forms a complex with integrin $\alpha v \beta 3$, an upstream regulator of AKT signaling, which indicated that AEP may regulate AKT signaling through integrins (32). The PI3K/AKT pathway is frequently activated during tumor progression and may be involved in inducing EMT and subsequent tumor metastasis $(38,39)$. Consistent with these reports, the present study demonstrated that the suppression of AEP expression significantly reduced AKT and PI3K phosphorylation. Further investigations are required to determine whether AEP may be a potential target for pancreatic adenocarcinoma treatment.

In conclusion, the present study identified the tumorpromoting functions of AEP in pancreatic adenocarcinoma and suggested that AEP may be a new target for the treatment of pancreatic adenocarcinoma; the discovery of novel therapeutic targets is important to improve the efficacy of pancreatic adenocarcinoma treatment.

\section{Acknowledgements}

Not applicable.

\section{Funding}

The present study was supported by The Zhejiang Science and Technology Plan Project (grant no. 2017C33189). 


\section{Availability of data and materials}

All data generated or analyzed during this study are included in this published article.

\section{Authors' contributions}

QY designed the study, acquired, analyzed and interpreted the data, and wrote the manuscript. WY, XS, MZ, FC, SZ, $\mathrm{WW}$ and YX collected and analyzed the data. YX, LT and ZM revised the manuscript.

\section{Ethics approval and consent to participate}

The present study was approved by the Ethics Committee of Huzhou Central Hospital, Zhejiang University (Huzhou, China). Written informed consent was obtained from patients, or from the guardians on behalf of the minors, prior to enrollment in the present study.

\section{Consent for publication}

Not applicable.

\section{Conflicts of interest}

The authors declare that they have no competing interests.

\section{References}

1. Jemal A, Bray F, Center MM, Ferlay J, Ward E and Forman D: Global cancer statistics. CA Cancer J Clin 61: 69-90, 2011.

2. Wood NJ: Pancreatic cancer: Pancreatic tumour formation and recurrence after radiotherapy are blocked by targeting CD44. Nat Rev Gastroenterol Hepatol 11: 73, 2014.

3. Liu P, Zhu Y and Liu L: Elevated pretreatment plasma D-dimer levels and platelet counts predict poor prognosis in pancreatic adenocarcinoma. Onco Targets Ther 8: 1335-1340, 2015.

4. Colbert LE, Hall WA, Nickleach D, Switchenko J, Kooby DA, Liu Y, Gillespie T, Lipscomb J, Kauh J and Landry JC: Chemoradiation therapy sequencing for resected pancreatic adenocarcinoma in the National Cancer Data Base. Cancer 120: 499-506, 2014.

5. Jagadeeshan S, Krishnamoorthy YR, Singhal M, Subramanian A, Mavuluri J,Lakshmi A, Roshini A, Baskar G, Ravi M, Joseph LD, et al: Transcriptional regulation of fibronectin by $\mathrm{p} 21$-activated kinase-1 modulates pancreatic tumorigenesis. Oncogene 34: 455-464, 2015

6. Ibrahim AM and Wang YH: Viro-immune therapy: A new strategy for treatment of pancreatic cancer. World J Gastroenterol 22: 748-763, 2016.

7. Al Haddad AH and Adrian TE: Challenges and future directions in therapeutics for pancreatic ductal adenocarcinoma. Expert Opin Investig Drugs 23: 1499-1515, 2014.

8. Haugen MH, Johansen HT, Pettersen SJ, Solberg R, Brix K, Flatmark K and Maelandsmo GM: Nuclear legumain activity in colorectal cancer. PLoS One 8: e52980, 2013.

9. Herskowitz JH, Gozal YM, Duong DM, Dammer EB, Gearing M, Ye K, Lah JJ, Peng J, Levey AI and Seyfried NT: Asparaginyl endopeptidase cleaves TDP-43 in brain. Proteomics 12: 2455-2463, 2012.

10. Miller G, Matthews SP, Reinheckel T, Fleming S and Watts C: Asparagine endopeptidase is required for normal kidney physiology and homeostasis. FASEB J 25: 1606-1617, 2011.

11. van Endert P: Toll-like receptor 9: AEP takes control. Immunity 31: 696-698, 2009

12. Choi SJ, Reddy SV, Devlin RD, Menaa C, Chung H, Boyce BF and Roodman GD: Identification of human asparaginyl endopeptidase (legumain) as an inhibitor of osteoclast formation and bone resorption. J Biol Chem 274: 27747-27753, 1999.
13. Zhen Y, Chunlei G, Wenzhi S, Shuangtao Z, Na L, Rongrong W, Xiaohe L, Haiying N, Dehong L, Shan J, et al: Clinicopathologic significance of legumain overexpression in cancer: A systematic review and meta-analysis. Sci Rep 5: 16599, 2015.

14. D'Costa ZC, Higgins C, Ong CW, Irwin GW, Boyle D, McArt DG, McCloskey K, Buckley NE, Crawford NT, Thiagarajan L, et al: TBX2 represses CST6 resulting in uncontrolled legumain activity to sustain breast cancer proliferation: A novel cancer-selective target pathway with therapeutic opportunities. Oncotarget 5: 1609-1620, 2014.

15. Sevenich L and Joyce JA: Pericellular proteolysis in cancer. Genes Dev 28: 2331-2347, 2014.

16. Edgington-Mitchell LE, Rautela J, Duivenvoorden HM, Jayatilleke KM, van der Linden WA, Verdoes M, Bogyo M and Parker BS: Cysteine cathepsin activity suppresses osteoclastogenesis of myeloid-derived suppressor cells in breast cancer. Oncotarget 6: 27008-27022, 2015.

17. Zhu W, Shao Y, Yang M, Jia M and Peng Y: Asparaginyl endopeptidase promotes proliferation and invasiveness of prostate cancer cells via PI3K/AKT signaling pathway. Gene 594: 176-182, 2016.

18. Hingorani SR: Intercepting Cancer Communiques: Exosomes as heralds of malignancy. Cancer Cell 28: 151-153, 2015.

19. Jørgensen M, Bæk R, Pedersen S, Søndergaard EK, Kristensen SR and Varming K: Extracellular Vesicle (EV) Array: Microarray capturing of exosomes and other extracellular vesicles for multiplexed phenotyping. J Extracell Vesicles 2: 2, 2013.

20. Hoshino A, Costa-Silva B, Shen TL, Rodrigues G, Hashimoto A, Tesic Mark M, Molina H, Kohsaka S, Di Giannatale A, Ceder S, et al: Tumour exosome integrins determine organotropic metastasis. Nature 527: 329-335, 2015.

21. Jeong D, Jo W, Yoon J, Kim J, Gianchandani S, Gho YS and Park J: Nanovesicles engineered from ES cells for enhanced cell proliferation. Biomaterials 35: 9302-9310, 2014.

22. Gao Q, Zhao YJ, Wang XY, Guo WJ, Gao S, Wei L, Shi JY, Shi GM, Wang ZC, Zhang YN, et al: Activating mutations in $P T P N 3$ promote cholangiocarcinoma cell proliferation and migration and are associated with tumor recurrence in patients. Gastroenterology 146: 1397-1407, 2014.

23. Kalluri R: The biology and function of exosomes in cancer. J Clin Invest 126: 1208-1215, 2016.

24. Vitale G, Zappavigna S, Marra M, Dicitore A, Meschini S, Condello M, Arancia G, Castiglioni S, Maroni P, Bendinelli P, et al: The PPAR- $\gamma$ agonist troglitazone antagonizes survival pathways induced by STAT- 3 in recombinant interferon- $\beta$ treated pancreatic cancer cells. Biotechnol Adv 30: 169-184, 2012.

25. Missiaglia E, Dalai I, Barbi S, Beghelli S, Falconi M, della Peruta M, Piemonti L, Capurso G, Di Florio A, delle Fave G, et al: Pancreatic endocrine tumors: Expression profiling evidences a role for AKT-mTOR pathway. J Clin Oncol 28: 245-255, 2010.

26. Stoll V, Calleja V, Vassaux G, Downward J and Lemoine NR: Dominant negative inhibitors of signalling through the phosphoinositol 3-kinase pathway for gene therapy of pancreatic cancer. Gut 54: 109-116, 2005.

27. Martini M, De Santis MC, Braccini L, Gulluni F and Hirsch E: PI3K/AKT signaling pathway and cancer: An updated review. Ann Med 46: 372-383, 2014.

28. Polivka J Jr and Janku F: Molecular targets for cancer therapy in the PI3K/AKT/mTOR pathway. Pharmacol Ther 142: 164-175, 2014.

29. Toren P and Zoubeidi A: Targeting the PI3K/Akt pathway in prostate cancer: Challenges and opportunities (review). Int $\mathbf{J}$ Oncol 45: 1793-1801, 2014.

30. Lin Y, Qiu Y, Xu C, Liu Q, Peng B, Kaufmann GF, Chen X, Lan B, Wei C, Lu D, et al: Functional role of asparaginyl endopeptidase ubiquitination by TRAF6 in tumor invasion and metastasis. J Natl Cancer Inst 106: dju012, 2014.

31. Qiu JJ, Lin YY, Ye LC, Ding JX, Feng WW, Jin HY, Zhang Y, $\mathrm{Li} \mathrm{Q}$ and Hua KQ: Overexpression of long non-coding RNA HOTAIR predicts poor patient prognosis and promotes tumor metastasis in epithelial ovarian cancer. Gynecol Oncol 134: 121-128, 2014.

32. Liu C, Sun C, Huang H, Janda K and Edgington T: Overexpression of legumain in tumors is significant for invasion/metastasis and a candidate enzymatic target for prodrug therapy. Cancer Res 63: 2957-2964, 2003.

33. Livak KJ and Schmittgen TD: Analysis of relative gene expression data using real-time quantitative PCR and the $2(-\Delta \Delta \mathrm{C}(\mathrm{T}))$ Method. Methods 25: 402-408, 2001. 
34. Kogure T, Lin WL, Yan IK, Braconi C and Patel T: Intercellular nanovesicle-mediated microRNA transfer: A mechanism of environmental modulation of hepatocellular cancer cell growth. Hepatology 54: 1237-1248, 2011.

35. Kamisawa T, Wood LD, Itoi T and Takaori K: Pancreatic cancer. Lancet 388: 73-85, 2016.

36. Chen JM, Dando PM, Rawlings ND, Brown MA, Young NE, Stevens RA, Hewitt E, Watts C and Barrett AJ: Cloning, isolation, and characterization of mammalian legumain, an asparaginyl endopeptidase. J Biol Chem 272: 8090-8098, 1997.

37. Wang L, Chen S, Zhang M, Li N, Chen Y, Su W, Liu Y, Lu D, $\mathrm{Li}$ S, Yang Y, et al: Legumain: A biomarker for diagnosis and prognosis of human ovarian cancer. J Cell Biochem 113: 2679-2686, 2012.
38. Mulholland DJ, Kobayashi N, Ruscetti M, Zhi A, Tran LM, Huang J, Gleave $\mathrm{M}$ and $\mathrm{Wu} \mathrm{H}$ : Pten loss and RAS/MAPK activation cooperate to promote EMT and metastasis initiated from prostate cancer stem/progenitor cells. Cancer Res 72: 1878-1889, 2012.

39. Bartolomé RA, García-Palmero I, Torres S, López-Lucendo M, Balyasnikova IV and Casal JI: IL13 Receptor $\alpha 2$ signaling requires a scaffold protein, FAM120A, to activate the FAK and PI3K pathways in colon cancer metastasis. Cancer Res 75: 2434-2444, 2015. 